\title{
Letter
}

\section{Universality of the quasinormal spectrum of near-extremal Kerr-Newman black holes}

\author{
Shahar Hod ${ }^{1,2, a}$ \\ ${ }^{1}$ The Ruppin Academic Center, 40250 Emeq Hefer, Israel \\ 2 The Hadassah Institute, 91010 Jerusalem, Israel
}

Received: 28 January 2015 / Accepted: 18 May 2015 / Published online: 23 June 2015

(C) The Author(s) 2015. This article is published with open access at Springerlink.com

\begin{abstract}
Our current knowledge about the quasinormal resonance spectrum of charged and rotating Kerr-Newman black holes is quite poor. This unsatisfactory situation is a direct consequence of the fact that all attempts to decouple the gravitational and electromagnetic perturbations of generic Kerr-Newman black holes have failed thus far. Recently, Zilhão et al. (Phys Rev D 90:12, 124088, 2014. arXiv:1410.0694) have studied the nonlinear stability of Kerr-Newman black holes. We show here that their numerical results for the time evolutions of the spacetime deformations of near-extremal Kerr-Newman black holes are described extremely well by a simple and universal analytical formula for the quasinormal resonances of the black holes. This formula is expressed in terms of the black-hole physical parameters: the horizon angular velocity $\Omega_{\mathrm{H}}$ and the Bekenstein-Hawking temperature $T_{\mathrm{BH}}$.
\end{abstract}

\section{Introduction}

It is well known that the fundamental quasinormal resonances of near-extremal black holes are described by an extremely simple and compact formula [1,2]:

$$
\omega_{n}=m \Omega_{\mathrm{H}}-i 2 \pi T_{\mathrm{BH}}\left(n+\frac{1}{2}-i \delta\right) ; \quad n=0,1,2, \ldots
$$

Here $^{1,2}$

$\Omega_{\mathrm{H}}=\frac{a}{r_{+}^{2}+a^{2}} \quad$ and $\quad T_{\mathrm{BH}}=\frac{r_{+}-r_{-}}{4 \pi\left(r_{+}^{2}+a^{2}\right)}$

\footnotetext{
1 We use natural units in which $G=c=\hbar=1$.

${ }^{2}$ Here $M, M a, Q$, and $r_{ \pm}=M \pm\left(M^{2}-a^{2}-Q^{2}\right)^{1 / 2}$ are, respectively, the mass, angular momentum, charge, and horizon-radii of the black hole.

a e-mail: shaharhod@gmail.com
}

are, respectively, the angular velocity of the black-hole horizon and the Bekenstein-Hawking temperature of the black hole, and $\delta$ is the angular-eigenvalue of the angular Teukolsky equation. ${ }^{3}$ The parameter $m$ in (1) is the azimuthal harmonic index of the perturbation mode.

It should be emphasized that the formula (1) was derived analytically $[1,2]$ for scalar, electromagnetic, and gravitational perturbations of rotating Kerr black holes. As for the charged and rotating Kerr-Newman black holes, this formula was formally derived only for scalar perturbation fields. The restriction to scalar fields in the case of Kerr-Newman black holes is a direct consequence of the fact that all attempts to decouple the gravitational and electromagnetic perturbations of generic Kerr-Newman black holes have failed thus far [4].

\section{Quasinormal oscillations of Kerr-Newman black holes}

Most recently, Zilhão et al. [5] have studied the nonlinear stability of Kerr-Newman black holes using fully nonlinear numerical simulations of the coupled Einstein-Maxwell equations. The numerical results presented in [5] indicate that, at late times, the spacetime deformations of the KerrNewman black hole are characterized by damped oscillations (see, in particular, Fig. 4 of [5]).

One of the most intriguing results presented in [5] is a numerical evidence for a universal behavior of these late-time spacetime oscillations (quasinormal resonances). In particular, Fig. 4 of [5] presents four time evolutions of quadrupolar spacetime deformations for four different values of the blackhole parameters $(a, Q)$, all share the same value of the ratio $a / a_{\max }=0.99$, where $a_{\max }=\sqrt{M^{2}-Q^{2}}$.

\footnotetext{
${ }^{3}$ The parameter $\delta$ is closely related to the angular-eigenvalue $\lambda$ of the angular Teukolsky equation; see [3] for details (see, in particular, Eqs. (2.7) and (6.3) of [3]).
} 
Three of these time evolutions, which correspond to the following Kerr-Newman parameters:

$(a / M, Q / M)=(0.907,0.4),(0.944,0.3),(0.99,0)$

overlap almost perfectly [5]. However, as indicated in [5], the curve describing the time evolution of the fourth perturbed black hole, which is characterized by

$(a / M, Q / M)=(0.594,0.8)$,

does not coincide with the previous three curves (time evolutions) [5].

Based on the (almost) perfect agreement between the time evolutions of the three perturbed Kerr-Newman black holes (3), it was suggested in [5] that the quadrupolar quasinormal resonances of Kerr-Newman black holes with large $a / Q$ ratios are characterized by a universal behavior of the form

$\omega=\omega\left(a / \sqrt{M^{2}-Q^{2}}\right)$.

That is, it was suggested [5] that the quasinormal resonances of Kerr-Newman black holes depend solely (and universally) on the ratio $a / a_{\max }$.

As discussed above, the almost identical temporal evolutions of the three perturbed black holes (3) are in agreement with the suggested universality (5). However, the time evolution of the fourth perturbed black hole (4), which does not coincide with the previous three time evolutions, raises doubts on the general validity of the suggested universal relation (5).

As we shall now show, all four temporal evolutions displayed in Fig. 4 of [5] [including the time evolution of the fourth black hole (4)!] are perfectly consistent with the formula (1). We first point out that the three black holes (3) are characterized by almost the same value of the horizon angular velocity $\Omega_{\mathrm{H}}$ (to an accuracy of better than $0.5 \%$ ) [see Eqs. (2) and (3)]

$\Omega_{\mathrm{H}} \simeq 0.432 \mathrm{M}^{-1}$

whereas the fourth black hole (4) is characterized by a different value of $\Omega_{\mathrm{H}}$

$\Omega_{\mathrm{H}} \simeq 0.388 \mathrm{M}^{-1}$

It is important to emphasize that time-dependent spacetime deformations, as the ones displayed in Fig. 4 of [5], consist of a sum of damped oscillations. Since, for nearextremal black holes, these oscillations (quasinormal resonances) share the same value of $\mathfrak{R} \omega$ [as suggested by (1)], one can accurately determine that value of $\Re \omega$ directly from the time evolutions presented in Fig. 4 of [5]. ${ }^{4}$

The quadrupolar ( $l=m=2$ ) spacetime oscillations of the three Kerr-Newman black holes (3) displayed in Fig. 4 of [5] are characterized by the (numerically computed) timeperiod $T_{\text {num }} \simeq 7.38 \mathrm{M}$. For comparison, the time-period $T_{\text {ana }}=2 \pi / \Re \omega=2 \pi / m \Omega_{\mathrm{H}}$ predicted by the analytical formula (1) is $T_{\text {ana }} \simeq 7.27 \mathrm{M}$ [see Eq. (6)]. One therefore finds a fairly good agreement (to better than $2 \%$ ),

$\frac{T_{\text {num }}}{T_{\text {ana }}} \simeq 1.015$,

between the analytical formula (1) and the numerical results of [5].

The quadrupolar spacetime oscillations of the fourth KerrNewman black hole (4) displayed in Fig. 4 of [5] are characterized by the (numerically computed) time-period $T_{\text {num }} \simeq 8.11 \mathrm{M}$. For comparison, the time-period $T_{\text {ana }}=$ $2 \pi / \Re \omega=2 \pi / m \Omega_{\mathrm{H}}$ predicted by the analytical formula (1) is $T_{\text {ana }} \simeq 8.09 \mathrm{M}$ [see Eq. (7)]. One therefore finds a remarkably good agreement (to better than $0.3 \%$ ),

$\frac{T_{\text {num }}}{T_{\text {ana }}} \simeq 1.003$

between the analytical formula (1) and the numerical results of [5].

\section{Summary}

In this brief report we have pointed out that the nonlinear (numerical) time evolutions of the near-extremal Kerr-Newman spacetime deformations presented in [5] are described extremely well by the simple analytical formula (1).

It is worth emphasizing again that the formula (1) was formally derived [1,2] for scalar, electromagnetic, and gravitational perturbations of near-extremal rotating Kerr black holes, as well as for scalar perturbations of near-extremal Kerr-Newman black holes. Formally, no analytical proof exists for its validity in the case of coupled gravitationalelectromagnetic perturbations of Kerr-Newman black holes.

However, the results presented here and in [5] strongly suggest that the formula (1) is actually of general validity. In

\footnotetext{
${ }^{4}$ On the other hand, the different overtones which compose the timedependent gravitomagnetic signals are characterized by different values of $\Im \omega_{n}$ [for different overtone numbers $n$, see Eq. (1)]. It is therefore difficult to determine accurately the various different values of $\Im \omega_{n}$ from the signals presented in Fig. 4 of [5] [as explained, these signals are 'contaminated' in the sense that they contain not one but several distinct overtones. Luckily, all of them share the same value of $\Re \omega$ (but not the same value of $\Im \omega)$ ].
} 
particular, we have shown that it accurately describes the coupled gravitational-electromagnetic quasinormal resonances of charged and rotating near-extremal Kerr-Newman black holes.

Finally, we would like to stress that the main body of the work was performed by the authors of [5]. It should be realized, however, that the novel (and important) numerical results presented in [5] cannot be properly understood (and, in particular, cannot be properly interpreted) without the crucial insight provided by the analytical formula (1). We therefore believe that the formula (1) may serve as an important guideline for future numerical and analytical explorations of the elusive (and poorly understood) quasinormal spectrum of charged and rotating Kerr-Newman black holes.

Acknowledgments This research is supported by the Carmel Science Foundation. I would like to thank Yael Oren, Arbel M. Ongo, Ayelet B. Lata, and Alona B. Tea for helpful discussions.
Open Access This article is distributed under the terms of the Creative Commons Attribution 4.0 International License (http://creativecomm ons.org/licenses/by/4.0/), which permits unrestricted use, distribution, and reproduction in any medium, provided you give appropriate credit to the original author(s) and the source, provide a link to the Creative Commons license, and indicate if changes were made.

Funded by $\mathrm{SCOAP}^{3}$.

\section{References}

1. S. Hod, Phys. Rev. D 78, 084035 (2008). arXiv:0811.3806

2. S. Hod, Phys. Lett. B 715, 348 (2012). arXiv: 1207.5282

3. S.A. Teukolsky, W.H. Press, Astrophys. J. 193, 443 (1974)

4. S. Chandrasekhar, The Mathematical Theory of Black Holes (Oxford University Press, Oxford, 1985)

5. M. Zilhão, V. Cardoso, C. Herdeiro, L. Lehner, U. Sperhake, Phys. Rev. D 90(12), 124088 (2014). arXiv:1410.0694 